\title{
The Effectiveness of Ellipsoidal Shape Representation Technique for 3D Object Recognition System
}

\author{
Asma Khatun, Wang Yin Chai, DNF Awang Iskandar, Md. Rabiul Islam \\ Universiti Malaysia Sarawak \\ Faculty of Computer Science and Information Technology \\ 94300, Kota Samarahan, Malaysia
}

\begin{abstract}
Shape representation methods play an important role in 3D shape recognition system. Three-dimensional shape recognition is widely used in 3D search engines, gravitational field, medical imaging, computer vision and face recognition. In this paper we propose an ellipsoidal shape representation technique for 3D shape recognition. We present some experimental and comparison results of our approach for shape matching using a standard database, Princeton Shape Benchmark. The effectiveness of our proposed algorithm is measured using nearest neighborhood. We then introduced a new idea which is a possible extension of the proposed approach and evaluate the results against human observation.
\end{abstract}

Keywords - 3D shape representation; recognition; ellipsoid mapping

\section{INTRODUCTION}

Three-dimensional (3D) shape recognition is relatively a new field in the domain of computer vision. It is a research study on how to recognize a 3D shape in a collection. As an emerging technology, 3D shape recognition continues to be one of the best research areas in computer vision and image understanding. One of the major challenges in 3D shape recognition is shape representation method. In order to achieve higher discrimination, there is a need to store important information of any 3D model. Hence, representing 3D shapes in an effective manner is one of the important principal criteria for a 3D shape recognition system.

Reference [1] stated that "shape representation methods result in a non-numeric representation of the original shape (e.g. a graph) so that the important characteristics of the shape are preserved. "In that paper it is also mentioned that shape descriptor is a numeric descriptor of the shape and is a step subsequent to shape representation. So it is clear that the requirement of shape representation method is very useful before processing for shape descriptor to collect the information. Survey papers on shape analysis have been provided by several authors such as [1-3]. In this paper we provide some study of shape representation methods for $3 \mathrm{D}$ shape recognition and its application. We also present experimental results of our proposed method [4] and compared with other existing methods using the nearest neighborhood. However in the literature spherical harmonic descriptor (SHD) and spherical wavelet descriptor (SWD) considered to be good performing descriptors. Surprisingly, we found that our proposed method [4] took the superior place despite the size of our descriptor is smaller (256b) than others such as SHD (2184b) and SWD (512b). The rest of the paper is organized as follows. In Section II, we present an overview of shape representation methods for 3D object recognition and its application. Section III describes the criteria for $3 \mathrm{D}$ shape representation methods. In section IV, we explain the use of ellipsoid as a mapping or projected function. In Section V, using the Princeton Shape Benchmark (PSB) we provide a comparison result using the nearest neighborhood. In Section VI, we present possible extension of our previous method and some observation results. Lastly, we conclude the paper with future works in section VII.

\section{CLASSIFICATION}

Currently most of the work on $3 \mathrm{D}$ object recognition places emphasis on geometrical attributes such as vertex, face, surface etc. $[5,6]$ and topological attribute such as graph, tree etc. $[7,8]$. A brief overview of different type of $3 \mathrm{D}$ object representation methods is provided by Funkhouser [9]. There are several applications of 3D shape recognition which implicates shape representation methods such as 3D search engines [10], molecular biology [11], astronomy and physics [12], 3D face recognition [13] and in the domain of computer vision. In this paper we concentrated more on 3D shape recognition in prospect of shape representation methods. We classified the existing work into two broad methods: geometry analysis and topology analysis.

\section{A. Geometry Analysis Based Methods}

In this method most of the work mapped with a function of 3D model to the particular domain. However most of the work used a spherical domain and has become one of the popular approaches to extract shape feature. 